\title{
MULTI-FREQUENCY VLBI OBSERVATIONS OF THE GRAVITATIONAL LENS B0218+357
}

\author{
R. W. PORCAS AND A. R. PATNAIK \\ Max-Planck-Intitut fuer Radioastronomie, Bonn, Germany
}

The gravitational lens system $\mathrm{B} 0218+357$ comprises 2 image components (A and B) and a radio 'Einstein Ring' (Patnaik et al, 1993). The redshift of the lens galaxy is 0.6847 (Browne et al, 1994) and that of the imaged source 0.96 (preliminary result; Lawrence et al, 1995). The separation of $\mathrm{A}$ and $\mathrm{B}$, which are both flat-spectrum radio sources, is only 0.335 arcsec, leading to the hope that the lens is a single galaxy with a relatively simple mass distribution. Refsdal pointed out (1964) that a model of such a distribution, and a measurement of the time difference along the two image paths, leads to an estimate of the Hubble constant, independent of the usual steps in the distance ladder. B0218+357 is one of only a few lensed systems well suited for such measurements. A preliminary value of 12 days has been measured for the A-B time delay, derived from a comparison of the percentage polarisation variations of the images at $15 \mathrm{GHz}$, using the VLA (Corbett et al, 1995).

We have made a detailed study of the mas structures of the $A$ and $B$ images, using a Global VLBI array at 1.6 and $5 \mathrm{GHz}$, and the VLBA at 8.4, 15, 22 and 43GHz (Patnaik et al, 1995, Porcas and Patnaik 1996). At $15 \mathrm{GHz}$ both the $\mathrm{A}$ and $\mathrm{B}$ images are resolved into two components, with a flux ratio between the components which is the same in both. The western component in each image is the more compact, and is identified as the "core". The components in image A (which is ca. 3.5 stronger than B at these frequencies) also exhibit extensions in $\mathrm{PA}-40^{\circ}$; this corresponds to the 'tangential stretching' expected from the geometry of this lens system. We have been able to deduce a relative magnification matrix between these images, and have also shown that the lens potential is non-spherical (Patnaik et al,1995).

At lower frequencies the compact cores become much weaker; both image structures are dominated by extended emission, which increases in size 

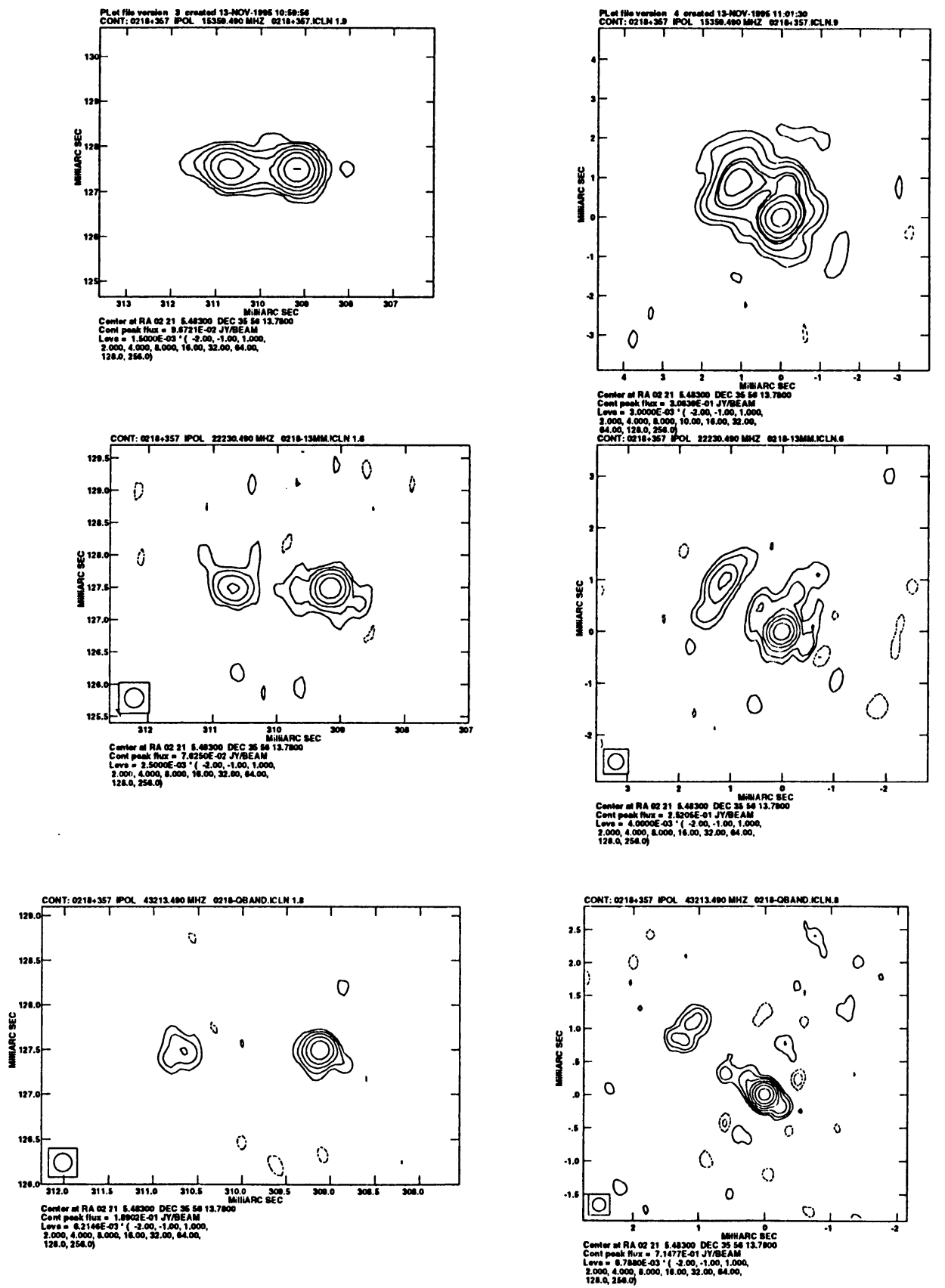

Figure 1. $15 \mathrm{GHz}$ (top), $22 \mathrm{GHz}$ (middle) and $43 \mathrm{GHz}$ (bottom) VLBA maps of B0218+357 made with resolutions $0.5,0.3$ and 0.2 mas, respectively, from observations on $17 \mathrm{July}, 1995$. The left panel shows image $B$ and the right image A. 
with wavelength, but maintains the same directions of extension visible in the higher frequency images. The flux ratio $A / B$ also decreases with wavelength. This can result when the image sizes are not small compared with the scale-size of the lens potential, resulting in gradients of magnification over the extended structure. Nair (1996) has made models of the B0218+357 system which take account of the fact that different parts of the background source are magnified quite differently.

Preliminary results from our latest VLBA observations at 15, 22 and 43 $\mathrm{GHz}$ are shown in Figure 1. At $15 \mathrm{GHz}$ the maps of $\mathrm{A}$ and B show structures very similar to our previous observations, with two prominent components in each image, and a northwards-pointing extension to the core in A, which has become more pronounced at this later epoch. At $22 \mathrm{GHz}$ and $43 \mathrm{GHz}$ both components are clearly seen in the $A$ and $B$ images, although the core becomes stronger at higher frequencies due to its inverted spectrum, and the northward extension in A (presumably steeper spectrum) becomes weaker. The other component maintains the characteristic elongation in A of PA $-40^{\circ}$ seen at $15 \mathrm{GHz}$; it is presumably this component which dominates in the maps of the structures at frequencies of $5 \mathrm{GHz}$ and less in both $\mathrm{A}$ and B.

\section{References}

Browne, I.W.A. et al, 1993, MNRAS,263, L32

Corbett, E. et al, 1996, Proc IAU Symp. 173,Astrophysical Applications of Gravitational Lensing, ed, Kochanek, C.S. and Hewitt, J.N., Kluwer, (in press)

Lawrence, C. et al,1996, Proc IAU Symp. 173,Astrophysical Applications of Gravitational Lensing, ed, Kochanek, C.S. and Hewitt, J.N., Kluwer, (in press)

Nair, S.,1996, Proc IAU Symp. 173,Astrophysical Applications of Gravitational Lensing, ed, Kochanek, C.S. and Hewitt, J.N., Kluwer, (in press)

Patnaik, A.R. et al, 1993, MNRAS, 261, 435

Patnaik, A.R., Porcas, R.W. and Browne, I.W.A., 1995, MNRAS, 274, L5

Porcas, R.W. and Patnaik, A.R., 1996, Proc IAU Symp. 173,Astrophysical Applications of Gravitational Lensing, ed, Kochanek, C.S. and Hewitt, J.N., Kluwer, (in press)

Refsdal, S., 1964, MNRAS, 123, 307 